\title{
Non-rigid Reconstruction of the Beating Heart Surface for Minimally Invasive Cardiac Surgery
}

\author{
Mingxing $\mathrm{Hu}^{1}$, Graeme P. Penney ${ }^{2}$, Daniel Rueckert ${ }^{3}$, Philip J. Edwards ${ }^{4}$, \\ Fernando Bello ${ }^{4}$, Roberto Casula ${ }^{5}$, Michael Figl ${ }^{3}$, and David J. Hawkes ${ }^{1}$ \\ ${ }^{1}$ Centre for Medical Image Computing, University College London \\ ${ }^{2}$ Department of Imaging Sciences, King's College London \\ ${ }^{3}$ Department of Computing, Imperial College \\ ${ }^{4}$ Department of Surgical Oncology and Technology, Imperial College \\ ${ }^{5}$ Cardiothoracic Surgery, St. Mary's Hospital, London, UK \\ \{mingxing.hu, d.hawkes\}@ucl.ac.uk, graeme.penney@kcl.ac.uk, \\ \{d.rueckert, eddie.edwards, f.bello,m.figl\}@imperial.ac.uk, \\ roberto.casula@st-mary.nhs.uk
}

\begin{abstract}
This paper presents a new method to reconstruct the beating heart surface based on the non-rigid structure from motion technique using preprocessed endoscopic images. First the images captured at the same phase within each heart cycle are automatically extracted from the original image sequence to reduce the dimension of the deformation subspace. Then the remaining residual non-rigid motion is restricted to lie within a low-dimensional subspace and a probabilistic model is used to recover the 3D structure and camera motion simultaneously. Outliers are removed iteratively based on the reprojection error. Missing data are also recovered with an Expectation Maximization algorithm. As a result the camera can move around the operation scene to build a 3D surface with a wide field-of-view for intra-operative procedures. The method has been evaluated with synthetic data, heart phantom data, and in vivo data from a da Vinci surgical system.
\end{abstract}

\section{Introduction}

Minimally invasive cardiac surgery (MICS) offers great benefits to patients, such as smaller incisions and less tissue trauma, shorter hospital stays and lower infection risk. Avoiding the use of the heart-lung machine leads to less bleeding and blood loss related trauma, so the patient can return to normal life more quickly than those who have conventional open surgery. Recently telemanipulator assisted techniques have been applied to MICS. These can enhance the manual dexterity of the surgeons and enable them to more fully concentrate on the surgical procedure. Despite all these advantages, MICS still suffers from a number of problems, such as: narrow field-ofview; restricted movement; and lack of 3D guidance information.

These problems have raised concerns from both research and clinical communities and work has been done to provide 3D information from intra-operative endoscopic video [1-5]. For example, Devernay et al. addressed a multiple-step strategy to achieve 3D reconstruction of the operative scene, in which they intended to reconstruct the organ surface from stereoscopy and then fuse these small patches from each 
camera position to construct a larger surface [1]. Then Lau et al. published a stereobased tracking method for cardiac surface deformation and used B-splines to model the heart surface to reduce the number of parameters to be computed [2]. Recently, Bader et al. also used stereo reconstruction to recover the 3D position of markers on a pulsating membrane to estimate the heart motion based on a state motion model [3]. However a drawback of these methods is that the images captured for $3 \mathrm{D}$ reconstruction are from a fixed field-of-view, so they cannot offer 3D information of peripheral scenes around the operating field. Recently Mountney et al. employed the SLAM (Simultaneous Location and Mapping) technique to build a 3D map of the scene and estimate the camera movement [4]. However, this requires long term repeatable landmarks to estimate the camera parameters, which are hard to obtain in dynamic scenes with soft tissue. More recently, $\mathrm{Hu}$ et al. proposed a new method to reconstruct internal organ surface based on the Structure from Motion (SFM) technique [5]. This can recover the $3 \mathrm{D}$ structure of the internal body from a moving endoscopic camera and so the reconstructed surface can cover a large area of the operation scene to provide a broader field-of-view for intraoperative procedures. However, this method does not allow for deformable objects and non-rigid modeling needs to be considered to improve accuracy and robustness.

This paper proposes a method to construct a heart surface from intra-operative endoscopic images of the beating heart. Our contributions are as follows. (i) The beating heart surface can be reconstructed with preprocessing using a non-rigid SFM technique. First, the beating heart cycle is robustly estimated using $\mathrm{Hu}$ 's algorithm based on geometric constraints [6]. Then the images captured at the same position of each cycle are selected for reconstruction in order to reduce the dimensionality of the deformation subspace. Then the deformable structure is restricted to lie within a lowdimensional subspace and a probabilistic model is used to recover the 3D structure and camera motion simultaneously. (ii) The endoscopic camera can move around the operation scene. Thus, the reconstructed surface can cover a large area of the operative scene, and it can provide the surgeon with a wider field-of-view including 3D information. (iii) Outliers are removed from the feature dataset using an iterative algorithm based on the reprojection error. Outliers obtained from bad locations and false tracking can disturb the reconstruction so much that the fitting parameters become arbitrary. To address this problem we compute the reprojection error of the features after each iteration, and remove the potential outliers.

\section{Methods}

\subsection{Pre-processing}

First we calibrate the endoscope in order to have more information about the internal properties of the camera, especially the distortion parameters. Endoscopes are often designed to have barrel distortion to capture a large field of view in a single image. Barrel distortion introduces nonlinear changes to the image and would introduce large errors to the 3D reconstruction. Here we use Zhang's planar calibration [7], which requires only a few images of a planar pattern from different unknown orientations. 
Because of the complexity of the cardiac motion, it is difficult to model it directly using simple deformable modeling techniques. To reduce the complexity of the heart motion for 3D reconstruction we re-arrange the endoscopic image sequence. We first analyze the heart motion based on geometric constraints using Hu's method and extract images from the same position of each heart cycle automatically for surface reconstruction. Usually we extract the images captured at the end-diastole, and for each heart cycle, two or three images are chosen. Then we employ a non-rigid modeling algorithm to model the residual cardiac motion remaining in the rearranged image sequence in order to reconstruct the heart surface. Thus we convert the difficult 4D (3D+time) dynamic reconstruction back to 3D reconstruction with small residual motion, which relatively is easier to solve. The electrocardiogram (ECG) signal could also be used to obtain the length of the heart cycle, however this requires extra hardware, an ECG machine, and synchronization between the endoscopic images and the ECG signal.

\subsection{Non-rigid Structure from Motion}

After the pre-processing, we employ the non-rigid structure from motion (NRSFM) [8-9] technique to reconstruct the beating heart surface using the re-arranged image sequence. Given a set of tracked feature points, the 2D and 3D locations can be related under weak-perspective projection as $[10,11]$

$$
\mathbf{x}_{i}^{j}=\lambda^{j} \mathbf{R}^{j}\left(\mathbf{X}_{i}^{j}+\mathbf{t}^{j}\right)
$$

where $\mathbf{x}_{i}^{j}=\left(x_{i}^{j}, y_{i}^{j}\right)^{T}$ is the projection of 3D point $\mathbf{X}_{i}=\left(X_{i}, Y_{i}, Z_{i}\right)^{T} \quad(i=1, \cdots, n)$ onto the $j$-th $(j=1, \cdots, \mathrm{m})$ image plane. $\mathbf{t}^{j}$ is a $3 \times 1$ translation vector, $\mathbf{R}^{j}$ is a $2 \times 3$ projection matrix, $\lambda^{j}$ is the weak-perspective scalar. The features tracked over $n$ frames are combined to form vectors

$$
\mathbf{x}^{j}=\mathbf{G}^{j}\left(\mathbf{X}^{j}+\mathbf{T}^{j}\right)+\mathbf{N}^{j}
$$

where $\mathbf{G}^{j}$ combines the matrix $\lambda^{j} \mathbf{R}^{j}$ and $\mathbf{N}^{j}$ is a noise vector added to the measurement vector $\mathbf{x}^{j}$. So the deformable shape can be represented in a K-dimensional linear subspace

$$
\mathbf{X}^{j}=\overline{\mathbf{X}}+\mathbf{V} \mathbf{z}^{j}+\mathbf{m}^{j}
$$

where $\mathbf{m}^{j}$ is the noise vector. Each column of the matrix $\mathbf{V}$ is a basis vector, and every entry of $\mathbf{z}^{j}$ is a corresponding weight that determines the contributions of the basis vector to the shape.

Torresani et al. proposed a method based on the probabilistic PCA (PPAC) model to estimate motion and learn the deformation basis [11]. The distribution of the deformable shapes is described as

$$
\mathbf{x}^{j} \sim \mathrm{N}\left(\mathbf{G}^{j}\left(\overline{\mathbf{X}}+\mathbf{T}^{j}\right) ; \mathbf{G}^{j}\left(\mathbf{V} \mathbf{V}^{T}+\sigma_{m}^{2} \mathbf{I}\right) \mathbf{G}^{j T}+\sigma^{2} \mathbf{I}\right)
$$

Then the EM algorithm is applied to estimate the PPCA model from feature points. That is, estimate the parameters $\mathbf{G}^{1: n}, \mathbf{T}^{1: n}, \overline{\mathbf{X}}$ and $\sigma^{2}$ from the given features $\mathbf{x}^{1: n}$. So the data likelihood can be described as

$$
p\left(\mathbf{x}^{1: n} \mid \mathbf{G}^{1: n}, \mathbf{T}^{1: n}, \overline{\mathbf{X}}, \mathbf{V}, \sigma^{2}\right)=\prod p\left(\mathbf{x}^{j} \mid \mathbf{G}^{j}, \mathbf{T}^{j}, \overline{\mathbf{X}}, \mathbf{V}, \sigma^{2}\right)
$$

The EM algorithm alternates between two steps: in the E step, a distribution over the latent coordinates $\mathbf{z}^{j}$ is computed; in the $\mathrm{M}$ step, the other variables are updated. As to the missing data $\mathbf{x}_{i^{\prime}}^{j^{\prime}}$ in the measurement matrix, they are also recovered during the M-step by optimizing the expected log likelihood. The reader can refer to [9] for more details. 


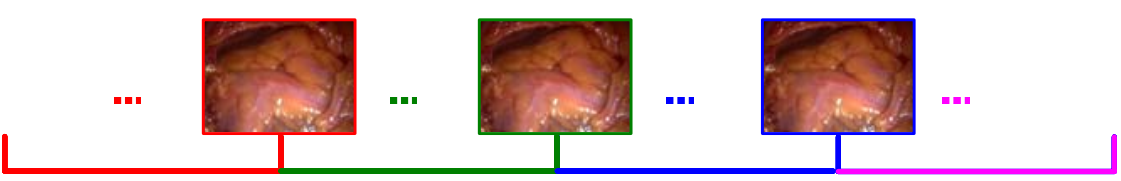

Fig. 1. Rearranged original image sequence. Images are extracted from the same phase in each beating heart cycle.

\subsection{Outlier Removal}

Outliers, which are caused by large errors in feature detection and tracking, are in gross disagreement with a specific postulated model and must be detected and removed from the feature database. Usually, outliers detected using epipolar geometry are features with a large geometric error (epipolar distance or Sampson distance). RANSAC (Random Sample Consensus Paradigm) is often employed as an outlier removal method [12]. However, because of the motion of the beating heart, there is still some residual motion in the rearranged image sequence obtained from Section 2.1. So the correspondences between the images do not satisfy the epipolar constraints. So rather than insisting on epipolar geometry, we use reprojection error to remove the outliers from the feature dataset.

After the estimation of the shape basis and the motion parameters, we reproject all the 3D points $\mathbf{X}_{i}(i=1, \cdots, m)$ to each image plane

$$
\hat{\mathbf{x}}_{i}^{j}=\lambda^{j} \mathbf{R}^{j}\left(\mathbf{X}_{i}^{j}+\mathbf{t}^{j}\right)=\lambda^{j} \mathbf{R}^{j}\left(\overline{\mathbf{X}}+\mathbf{V} \mathbf{z}^{j}+\mathbf{t}^{j}\right)
$$

where $\hat{\mathbf{x}}_{i}^{j}$ is the reprojection of point $\mathbf{X}_{i}$ on image $j(j=1, \cdots, n)$. Then we construct a weight matrix $\mathbf{W}$ of size $n \times m$, in which 0 s correspond to missing elements, and $1 \mathrm{~s}$ to the others.

So the RMS error between the reprojection $\hat{\mathbf{x}}_{i}^{j}$ and the measurement $\mathbf{x}_{i}^{j}$ for point $\mathbf{X}_{i}$ can be denoted as

$$
E_{i}=\sum_{j=1}^{n} w_{i}^{j} \cdot\left\|\hat{\mathbf{x}}_{i}^{j}-\mathbf{x}_{i}^{j}\right\|_{F}^{2} / \sum_{j=1}^{n} w_{i}^{j}
$$

where \|\|$_{F}$ is the Frobenius norm. Then the decision rule for inliers and outliers can be expressed as

$$
\begin{cases}\text { inlier } & \text { if } E_{i} \leq \gamma \\ \text { outlier } & \text { otherwise }\end{cases}
$$

$\gamma=1.96 \sigma$ is the threshold for considering the inliers, and the standard deviation $\sigma$ can be found as a maximum likelihood estimate using the median $\sigma=1.4828(1+5 /(n-7)) \sqrt{\text { med }_{i}\left|E_{i}\right|}$. An overview of the complete algorithm is given in Fig. 2

\subsection{Experimental Design}

In order to assess the performance of the proposed method, a beating heart phantom made of silicone rubber (The Chamberlain Group, Great Barrington, MA, USA) was employed to provide a gold standard for evaluation. The phantom is continuously inflated and deflated using an air pump with an integrated controller. In order to create a beating heart model, it was scanned while beating at the rate of $55 \mathrm{bpm}$ with a Philips 64-slice CT scanner, producing 10 uniformly-spaced phases. 
Input: Endoscopic image sequence of the beating heart

Output: 3D shape and motion parameters

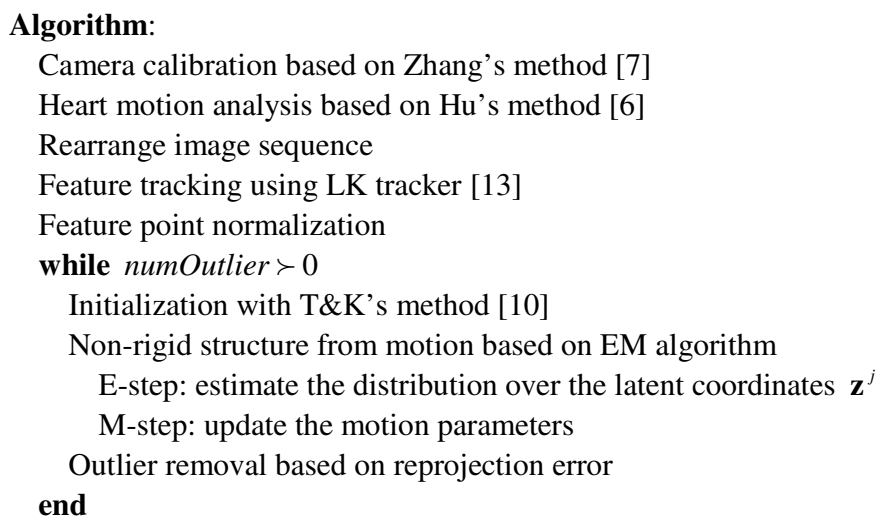

Fig. 2. Non-rigid reconstruction of the beating heart surface

The first of these was manually segmented and converted into a tetrahedral mesh of 709 elements and 747 degrees of freedom. The Image Registration Toolkit [14] was then used to create a sequence of 3D tensor product cubic B-splines deformations, mapping the initial mesh onto each phase in turn. Fig. 3 (a) and (b) shows the heart phantom and resulting mesh respectively. The heart phantom was also scanned without beating and a 3D model was created using the marching cubes algorithm as shown in Fig. 3 (c).

We have carried out experiments on three sets of data: synthetic test data, heart phantom data and in vivo data from endoscopic surgery. These sets of data and a description of the experiments are described in the following paragraphs.

In synthetic data testing, 100 points were randomly selected from the beating heart model described in Section 2.4. For simplicity, we adjust the beating heart rate to $60 \mathrm{bpm}$ (beats per minute). Then a moving camera was simulated with frame rate $25 \mathrm{fps}$, and all these 3D dynamic points were projected to 50 image planes with different rotation and translation parameters to generate sets of $2 \mathrm{D}$ image points. Instead of rearranging the image sequence, we used all the images created from different positions of the heart cycle for 3D reconstruction. So the 50 images included two heart cycles.

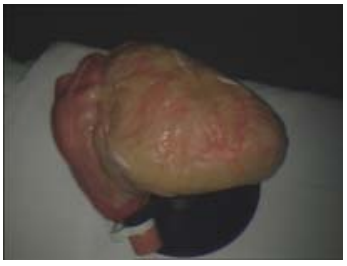

(a)

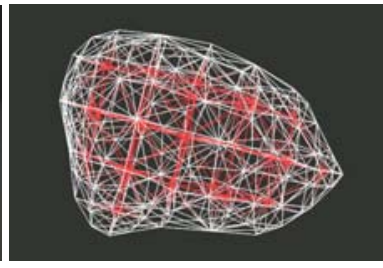

(b)

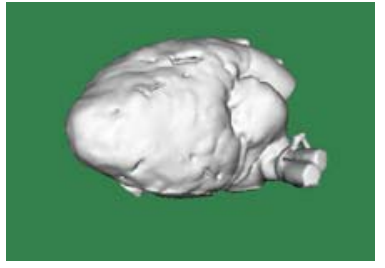

(c)

Fig. 3. Heart phantom. (a) Image of the heart phantom; (b) FEM mesh from CT data of the beating phantom; (c) 3D model from CT data of the phantom without beating. 
The $d a$ Vinci $^{\mathrm{TM}}$ robotic system (Intuitive Surgical, Inc., Sunnyvale, CA, USA) was used to obtain images of the heart phantom. The beating heart rate was set to $75 \mathrm{bpm}$ and the endoscopic camera was moved around the scene. 250 images were captured with the frame rate $25 \mathrm{fps}$ and the first and last frames are shown in Fig. 5 (a) and (b) respectively.

The in vivo data testing consisted of endoscopic images collected from a TECAB (Totally Endoscopic Coronary Artery Bypass) surgical procedure. Fig. 6 (a) and (b) show the first and last frames respectively. The recorded ECG signal was used as ground truth for heart motion analysis, which was $19.7 \mathrm{fpc}$ (frame per cycle).

\section{Experimental Results}

\subsection{Synthetic Data}

10 different ranges of Gaussian noise were added to the image measurements, with a zero mean and standard deviation varying from 0.5 to 5.0 pixels. In addition, $20 \%$ of the image points were occluded for some fraction to simulate missing data. For each noise level, we ran 200 trials and the final results were the average of results from these 200 independent experiments. A graph of these results is shown in Fig. 4 (a). It can be noticed that the RMS error of the proposed method increases gradually with the increase of noise. As the problem becomes more difficult, i.e., corrupted with severe noise ( $\sigma \geq 3.5$ pixels), the error increases more quickly.

We also investigated the RMS error under different number of basis shapes as shown in Fig. 4 (b). The error decreases as the dimension $\mathrm{K}$ increases but it reduces more slowly when the dimension $\mathrm{K}$ is bigger than 4 . All these indicate the proposed method can obtain an accurate result and is robust to missing data and the choice of $\mathrm{K}$ due to the implementation of the probabilistic PCA model and EM algorithm.

\subsection{Heart Phantom Data}

The first 100 images were employed to estimate the heart cycle using Hu's method [6]. The experimental result was $20.2 \mathrm{fpc}$ (frame per cycle), which is close to the ground truth $(60 / 75) * 25=20 \mathrm{fpc}$. Because the estimated result is a real value, for each heart cycle, the two images closest to the end-diastole were chosen. So 20 images were extracted

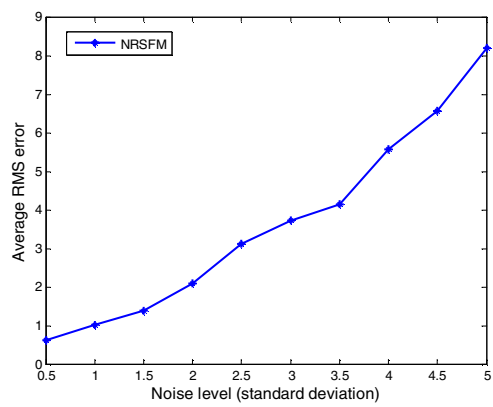

(a)

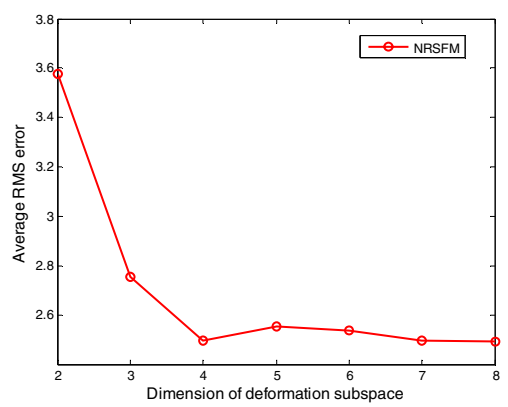

(b)

Fig. 4. Experimental results of synthetic data testing. (a) Average RMS error under different noise level; (b) Average RMS error under different dimension of deformation subspace (K). 


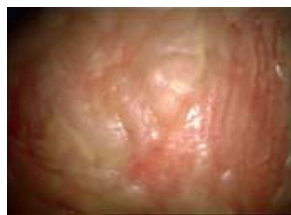

(a)

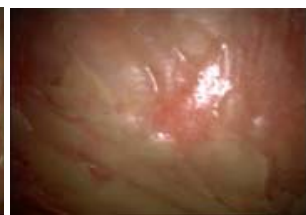

(b)

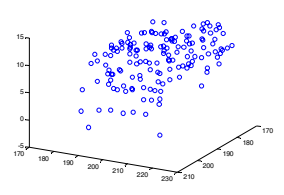

(c)

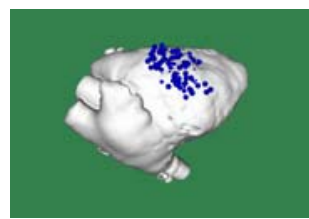

(d)

Fig. 5. Results using the heart phantom data. (a) and (b) show the first and last frames of the image sequence of phantom heart respectively; (c) and (d) display the reconstructed point cloud and its registration result to $\mathrm{CT}$ model.

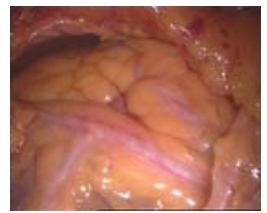

(a)

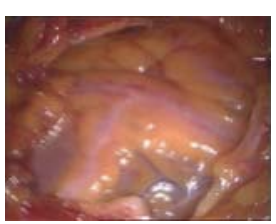

(b)



(c)

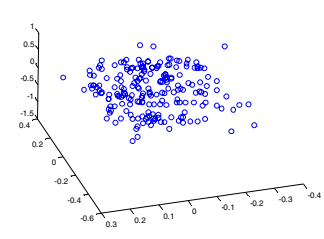

(d)

Fig. 6. Experimental results of in vivo data testing. (a) and (b) show the first and last frames of the image sequence from TECAB surgery; (c) illustrate the data filling results of a feature; (d) displays the reconstructed point cloud using the proposed method.

from the first ten heart cycles to generate the rearranged image sequence. Then the LK feature tracker was used to track 236 feature points. The missing data rate was $27.3 \%$ because some features moved out of the scene due to the movement of the camera. Fig. 5 (c) displays the reconstructed 3D point cloud using the proposed method. Since we did not have a gold standard transformation between the 3D points and our CT image, we used the Iterative Closet Point (ICP) [15] algorithm to register the $3 \mathrm{D}$ points to the surface derived from $\mathrm{CT}$ to obtain a measure of $3 \mathrm{D}$ reconstruction accuracy. Fig. 5 (d) shows the final position after ICP registration. Most of the points lie on or are close to the surface and the mean residual is around $3.7 \mathrm{~mm}$. Moreover, using visual inspection we can see that the positions of 3D points are overlaid on the CT surface after ICP registration in roughly the correct position.

\subsection{In vivo Data}

The estimated heart rate was $19.6 \mathrm{fpc}$ using Hu's method. Then 20 images were extracted from 10 heart cycles based on the estimated result. 215 features were detected and tracked from the rearranged 20 images and on average $21.1 \%$ of data in the measurement matrix was missing. Fig. 6(c) shows the results of our missing data filling algorithm on the in vivo data. The measured features are marked with blue "o" and the reprojected points to those image planes are marked with red " $x$ ". The feature point shown was detected and tracked until the $16^{\text {th }}$ frame, however our method was able to recover its position accurately in frames 17 and 18 .

Since no ground truth data was available for this patient, we report the RMS error of the surface reconstruction instead. Before removing the outliers, the average RMS error for all the visible features was $2.9 \mathrm{~mm}$. This reduced to $2.4 \mathrm{~mm}$ after 16 outliers 
were detected and removed from the feature dataset. Fig. 6 (d) shows the point cloud reconstructed from the endoscopic image sequence.

\section{Discussion and Conclusions}

This paper proposes a non-rigid reconstruction of the beating heart surface for MICS. The original image sequence is rearranged to reduce the dimension of deformable subspace of the heart surface. Then a non-rigid structure-from-motion technique is applied to recover the 3D dynamic structure and camera motion simultaneously. Outliers are removed iteratively based on the reprojection error and missing data are recovered with the EM algorithm.

Effort in the near future will focus on creating a series of 3D heart surfaces from different phases of the heart cycle to generate the whole dynamic heart surface. Incorporating a more sophisticated deformable model, e.g. a bio-mechanical model, for the non-rigid SFM is also an interesting direction. Currently it takes minutes to finish the whole estimation (pre-processing and non-rigid reconstruction), which could be a big problem to clinical utility. So we are going to use more efficient algorithms and Graphical Processing Unit (GPU) technology to achieve real-time computation. Our long term goal is to automatically reconstruct the surfaces of internal organs and register these with the preoperative data (CT or MRI) to provide more information for intra-procedural targeting, monitoring and control.

Acknowledgments. The work was supported by the UK Engineering and Physical Sciences Research Council (EPSRC) under Grant No. EP/C523016/1, and EPSRC Advanced Research Fellowship GR/T02799/02 for GPP.

\section{References}

1. Devernay, F., Mourgues, F., Coste-Maniere, E.: Towards Endoscopic Augmented Reality for Robotically Assisted Minimally Invasive Cardiac Surgery. In: Proc. International Workshop on Medical Imaging and Augmented Reality, pp. 16-20 (2001)

2. Lau, W.W., Ramey, N.A., Corso, J.J., Thakor, N.V., Hager, G.D.: Stereo-Based Endoscopic Tracking of Cardiac Surface Deformation. In: Barillot, C., Haynor, D.R., Hellier, P. (eds.) MICCAI 2004. LNCS, vol. 3217, pp. 494-501. Springer, Heidelberg (2004)

3. Bader, T., Wiedemann, A., Roberts, K., Hanebeck, U.D.: Model-Based Motion Estimation of Elastic Surfaces for Minimally Invasive Cardiac Surgery. In: Proc. ICRA, pp. 22612266 (2007)

4. Mountney, P., Stoyanov, D., Davison, A.J., Yang, G.-Z.: Simultaneous Stereoscope Localization and Soft-Tissue Mapping for Minimal Invasive Surgery. In: Larsen, R., Nielsen, M., Sporring, J. (eds.) MICCAI 2006. LNCS, vol. 4190, pp. 347-354. Springer, Heidelberg (2006)

5. Hu, M., Penney, G.P., Edwards, P., Figl, M., Hawkes, D.J.: 3D Reconstruction of Internal Organ Surfaces for Minimal Invasive Surgery. In: Ayache, N., Ourselin, S., Maeder, A. (eds.) MICCAI 2007, Part I. LNCS, vol. 4791, pp. 68-77. Springer, Heidelberg (2007) 
6. Hu, M., Penney, G.P., Rueckert, D., Edwards, P., Figl, M., Pratt, P., Hawkes, D.J.: A Novel Algorithm for Heart Motion Analysis Based on Geometric Constraints. In: Metaxas, D., Axel, L., Fichtinger, G., Székely, G. (eds.) MICCAI 2008, Part I. LNCS, vol. 5241, pp. 720-728. Springer, Heidelberg (2008)

7. Zhang, Z.: A Flexible New Technique for Camera Calibration. IEEE Trans. PAMI 22(11), 1330-1334 (2000)

8. Torresani, L., Hertzmann, A., Bregler, C.: Learning Non-Rigid 3D Shape from 2D Motion. In: Proc. NIPS, pp. 1555-1562 (2004)

9. Torresani, L., Hertzmann, A., Bregler, C.: Nonrigid Structure-from-Motion: Estimating Shape and Motion with Hierarchical Priors. IEEE Trans. PAMI 30(5), 878-892 (2008)

10. Tomasi, C., Kanade, T.: Shape and Motion from Image Streams under Orthography: a Factorization Method. Int. J. Computer Vision 9(2), 137-154 (1992)

11. Bregler, C., Hertzmann, A., Biermann, H.: Recovering Non-Rigid 3D Shape from Image Streams. In: Proc. CVPR, pp. 690-696 (2000)

12. Fischler, M.A., Bolles, R.C.: Random sample consensus: a paradigm for model fitting with applications to image analysis. Comm. ACM 24, 381-385 (1981)

13. Lucas, B., Kanade, T.: An Iterative Image Registration Technique with an Application to Stereo Vision. In: Proc. IJCAI, pp. 674-679 (1981)

14. Image registration toolkit, http://www. doc.ic.ac.uk/ dr/software/

15. Besl, P., McKay, N.: A Method for Registration of 3-D Shapes. IEEE Trans. PAMI 14(2), 239-256 (1992) 